\title{
Origen botánico y contenido en proteína de mieles artesanales procedentes de colmenares de Galicia (NO de España)
}

\author{
Judit Rodríguez-Castiñeira, Sandra Armesto-Baztán y Ma Pilar de Sá-Otero ${ }^{1}$
}

\begin{abstract}
Resumen: Rodríguez-Castiñeira, J.; Armesto-Baztán, S. \& de Sá-Otero, M. P. 2015. Origen botánico y contenido en proteína de mieles artesanales procedentes de colmenares de Galicia (NO de España). Bot. Complut. 39: 105-114.

Se ha estudiado el origen botánico y contenido en proteína de quince mieles producidas en pequeñas explotaciones, para consumo familiar, en Galicia, con el fin de ver si riqueza proteica y polínica son parámetros dependientes. Han resultado siete monoflorales de Rhamnus frangula (de baja diversidad de su espectro polínico), dos monoflorales de Castanea sativa Miller, una monofloral de brezo y cinco mieles milflores diversas en su espectro polínico ( 4 milfores de Castanea sativa. predominante y 1 milflores de Rhamnus frangula predominante). Su riqueza polínica ha sido baja, ocho mieles clasificadas en la clase I de Maurizio, tres, clase Maurizio II, dos, clase Maurizio III y una, clase Maurizio IV. Se obtuvo una amplia variabilidad en su contenido en proteína (0,09-4,83 $\mathrm{mg}$ prot./g miel). La cantidad relativa de polen de los diferentes taxones tiene una relación de proporcionalidad directa 0 inversa con la riqueza en proteína.
\end{abstract}

Palabras clave: espectro polínico, riqueza proteica, riqueza polínica, monofloral de Rhamnus frangula.

Abstract: Rodríguez-Castiñeira, J.; Armesto-Baztán, S. \& de Sá-Otero, M. P. 2015. Botany origin and protein content of homemade honeys from Galicia hives (NW Spain). Bot. Complut. 39: 105-114.

The botanic origin and the protein content of 15 honeys from small bee farms exploitations of Galicia, for family consume, were studied; the aim is to check if the protein wealth and the pollen wealth are dependent parameters. Seven honeys resulted to be Rhamnus frangula unifloral (pollen patterns with low diversity), two Castanea sativa Miller unifloral, other one heather unifloral, and five was multifloral honeys of various pollen patterns (four Castanea predominant and one Rhamnus frangula predominant). Their pollen wealth was low; eight honeys classified in the Maurizio Class I, 3 in Class II, 2 in Class III, and one in Maurizio Class IV. There has been a wide variability in its protein content $(0.09$ $4.83 \mathrm{mg}$ prot./g honey). The relative amount of pollen from different taxa has a direct or inverse proportionality to wealth protein.

Key words: pollen pattern, protein wealth, pollen wealth, Rhamnus frangula, unifloral.

\section{INTRODUCCION}

Las principales fuentes para la elaboración de la miel son el néctar y el mielato, ambos recogidos por las abejas en las plantas y sometidos por éstas a procesos enzimáticos que conducen a la formación de un producto natural, con alto valor biológico y funcional. Se oferta al consumidor prácticamente sin procesado industrial.

El contenido en proteínas de las mieles es una consecuencia directa del contenido polínico de las mismas, tanto de su riqueza polínica (número de granos de polen por gramo de miel), como de la diversidad de tipos polínicos contenidos en las mismas (Crane 1975). Algunos autores como Gheldof et al. (2002) han establecido una relación de proporcionalidad positiva entre la capacidad antioxi- dante de las mieles y su contenido en proteínas y compuestos fenólicos.

Fonseca et al. (1993) han establecido diferentes contenidos de proteínas en mieles de diferente origen floral. Así, en mieles de azahar hallan valores de 0,85-2,62 g prot./100 g miel, cuyo valor medio es de $1,4 \mathrm{~g}$ prot./100 g miel. En las mieles de eucalipto el rango se establece de $1,0-2,26 \mathrm{~g}$ prot./100 g miel, su valor medio es de $1,84 \mathrm{~g}$ prot./100 g miel. Estos autores establecen, además, que la cantidad relativa de proteína varía en relación a la naturaleza del polen dominante, y también en función de la naturaleza de las especies encontradas en el espectro en proporciones menores. Otras mieles, de origen floral diverso, también analizadas por éstos, han dado valores cuyo máximo es de $3,2 \mathrm{~g}$ prot./100 g miel.

\footnotetext{
${ }^{1}$ Departamento de Bioloxía Vexetal e Ciencia do Solo, Facultad de Ciencias de Ourense, Universidade de Vigo, España saa@uvigo.es; sandrabaztan@yahoo.es, judiguer@hotmail.com

Recibido: 17 julio 2014. Aceptado: 13 octubre 2014
} 
Baroni et al. (2002) relacionaron positivamente el origen monofloral de las mieles y la presencia de proteínas específicas en las mismas. Azedero et al. (2003) establecen también relaciones directas entre el origen floral de las mieles y su contenido proteico. Cremonez et al. (1998) consideran el polen como fuente de proteínas en la colmena y logran establecer una relación directa entre la proteína contenida en la hemolinfa de las abejas y el aporte proteico que entra en la colmena, a través del análisis del polen corbicular traído por las abejas a la colmena. De Sá-Otero et al. (2009) han obtenido que el polen de Cytisus scoparius-t., gramínea silvestre, Quercus robur-t., Sanguisorba minor, Salix fragilis y Spergularia rubra tiene altos porcentajes relativos de proteína, estos pólenes son frecuentes en el espectro polínico de las mieles gallegas. Por otra parte, Padovan et al. (2003) han utilizado la presencia de proteínas en la miel como fuente de carbono para establecer la relación de ${ }^{13} \mathrm{C} /{ }^{12} \mathrm{C}$, como método de detección de fraude en el de origen botánico de las mismas.

El presente trabajo se trata de estudiar la posible relación entre el contenido en proteína y la riqueza polínica de las mieles, además de la influencia de la naturaleza del espectro polínico en el valor de dicho parámetro.

\section{MATERIALES Y MÉTODOS}

Se ha hecho análisis polínico cualitativo y cuantitativo de 15 muestras de miel artesanal, producidas en Galicia, en colmenares de pequeña producción, para uso familiar, situados en diversos lugares de la comunidad autónoma gallega (Fig. 1). Son mieles de una única extracción anual, obtenidas por centrifuga- do, en los meses de agosto y septiembre de 2010. Para el análisis polínico porcentual, la metodología de extracción de polen en miel ha sido la propuesta por Loveaux et al. (1978), partiendo de $20 \mathrm{~g}$ de cada una de las mieles. Para el análisis porcentual se ha procedido según la metodología propuesta por Sá-Otero et al. (1993), leyendo cuatro preparaciones microscópicas por muestra de miel. En cada una de ellas se identificaron la cuarta parte del total de pólenes a partir de los que se hizo el cálculo de porcentajes. Las preparaciones microscópicas se han confeccionado sobre glicerina fenolada, sin tinción.

Como material de referencia para la identificación polínica, se han utilizado preparaciones microscópicas, elaboradas a partir del polen de plantas recogidas en los alrededores de los colmenares artesanales, la palinoteca existente en el laboratorio de Palinología Aplicada y Viticultura de la Facultad de Ciencias de Ourense y los tratados de Palinología de Valdés et al. (1987), Moore et al. (1991) y Sá-Otero et al. (1996). Las identificaciones polínicas se han hecho a nivel de especie, siempre que ha sido posible, familia, género o tipo polínico. El tipo polínico incluye especies $\mathrm{y} / \mathrm{o}$ géneros presentes en una misma área geográfica que poseen la misma forma polínica. Para el análisis cuantitativo se ha empleado una Neubauer Conting Cell. Los resultados se expresan en forma de clases de frecuencia Maurizio (1939): clase I, <2.000 granos de polen/g miel; clase II, entre 2.000-10.000; clase III, entre 10.000-50.000; clase IV, entre $50.000-100.000$ y clase $\mathrm{V}>100.000$.

La determinación de proteínas se ha realizado por el método de Bradford (1976), consistente en la tinción (unión específica de una sustancia coloreada, reactivo Bradford, a la proténa) y medida por espectrofotometría (a $595 \mathrm{~nm}$ ). Para la preparación del reactivo Bradford se pesan $100 \mathrm{mg}$ de Coomasie G-250 y se enrasa a un volumen de $50 \mathrm{~mL}$, con etanol al $95 \%$. A esta solución se le añade ácido ortofosfórico, enrasándola hasta $100 \mathrm{~mL}$. Se agita hasta su completa disolución y finalmente se le añade agua destilada hasta un volumen final de $1.000 \mathrm{~mL}$.

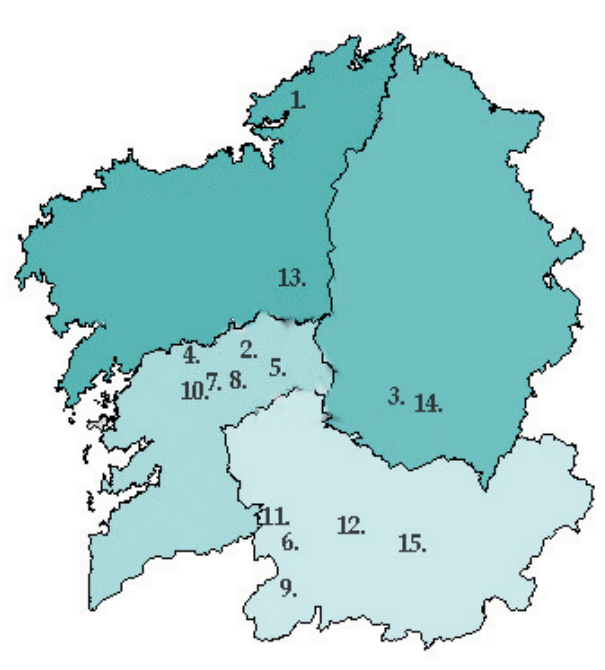

\begin{tabular}{cll}
$\begin{array}{c}\mathbf{N}^{\mathbf{0}} \\
\text { muestra }\end{array}$ & \multicolumn{1}{c}{ Localidad } & \multicolumn{1}{c}{ Ayuntamiento } \\
\hline 1 & Olmo & Ferrol \\
2 & Cereixo & A Estrada \\
3 & Currelos & O Saviñao \\
4 & Eixón & A Estrada \\
5 & Vilatuxe & Lalín \\
6 & Milmanda & Celanova \\
7 & Vistalegre & Lalín \\
8 & Soutolongo & Lalín \\
9 & Guntumil & Muíños \\
10 & Zobra & Lalín \\
11 & Carballeda de Avía & Carballeda de Avía \\
12 & Balias & San Cristovo de Cea \\
13 & Barciela & Santiago de Compostela \\
14 & Neiras & Sober \\
15 & A Costa & Maceda \\
\hline
\end{tabular}

Fig. 1- Mapa de localización de las mieles estudiadas. 
Se reserva en la oscuridad hasta su posterior utilización. Para la preparación de la proteína de referencia (seroalbúmina bovina) se pesan $10 \mathrm{mg}$ de seroalbúmina bovina y se disuelven en $10 \mathrm{~mL}$ de agua destilada. La disolución final se divide en alícuotas de $0,5 \mathrm{~mL}$ y se reservan en el congelador. Para la preparación de la muestra problema se disuelven $2,5 \mathrm{~g}$ de miel en $50 \mathrm{~mL}$ de agua destilada. A continuación y en agitación continua, se procede a elevar su pH hasta 8,5, con $\mathrm{NaOH}$. La recta de calibrado se obtiene a partir de una serie de disoluciones de la proteína patrón a distintas concentraciones, todas ellas con un volumen final de $100 \mu \mathrm{L}$. Posteriormente, se añaden $2 \mathrm{~mL}$ del reactivo Bradford a cada disolución, con el fin de que éste no sea el reactivo limitante en el proceso de unión tinte-proteína. Cada una de estas cubetas reflejará una absorción de luz diferente y proporcional a la concentración de proteína disuelta; lo que proporcionará una recta patrón a la que referir los datos de las muestras problema. La absorbancia se mide a una longitud de onda de $595 \mathrm{~nm}$.

La cuantificación de la proteína presente en la muestra problema se lleva a cabo preparando disoluciones de la muestra problema de 40 y $80 \mu \mathrm{L}$, enrasando a $100 \mu \mathrm{L}$ con $\mathrm{H}_{2} \mathrm{O}$ destilada. A cada disolución se le añaden $2 \mathrm{~mL}$ del reactivo Bradford y se procede a leer la absorbancia a $595 \mathrm{~nm}$. Este proceso se realiza por triplicado. El valor medio de la absorbancia obtenido para las tres réplicas, permite, en relación a la recta patrón, la determinación de la proteína presente en la muestra problema.

\section{RESULTADOS Y DISCUSIÓN}

Origen floral. Las mieles han resultado ser: siete, mieles monoflorales de Rhamnus frangula (sanguiño) (mues$\operatorname{tras~}^{0} 1,4,6,9,12,14$ y 15); dos, mieles monoflorales de Castanea sativa (castaño) (muestras 7 y 8), y una miel monofloral de Erica $\left(\mathrm{n}^{0} 2\right)$. Las 5 muestras restantes son mieles milflores con predominio de Castanea sativa $\left(\mathrm{n}^{0} 3,5\right.$, 10 y 13) y de Rhamnus (11). En el análisis polínico cualitativo se han identificado 26 tipos polínicos, pertenecientes a 16 familias distintas (Tabla.1). De estos, 14 se hallaron en porcentajes muy bajos (menores del $3 \%$ ). La diversidad del espectro ha sido diferente para las distintas mieles. Las muestras 9 y 13 han sido las más diversas, con 12 tipos polínicos, y la muestra $\mathrm{n}^{0} 14$ ha sido la menos diversa, reúne sólo polen de 3 fuentes de néctar (Tabla 1). Los tipos polínicos más frecuentes y de mayor representatividad han sido Rubus ulmifolius-t., Rhamnus frangula y Castanea sativa (Fig. 2). En cuanto a la riqueza polínica, las muestras analizadas han resultado tener un bajo número de granos de polen por gramo de miel, solo una de ellas ha alcanzado un valor medio-alto (muestra $\mathrm{n}^{0} 14$ ) (Tabla 1). El valor de proteína en las mieles varió desde $0,086 \mathrm{mg}$ prot./g miel y $4,825 \mathrm{mg}$ prot./g miel, con una media de $2,168 \mathrm{mg}$ prot./g miel (Tabla 1). Por su origen floral, las mieles de mayor riqueza (2,843 $\mathrm{mg}$ prot./g miel) han sido las de Castanea dominante, seguidas de las milflores (2,6 $\mathrm{mg}$ prot./g miel). Las de Rhamnus dominante son de riqueza media (2,056 mg prot./g miel) y la única miel monofloral de Erica presenta una riqueza de 1,6 mg prot/g miel.

Las mieles. Es destacable la presencia de mieles monoflorales de Rhamnus frangula, que si bien han sido descritas en otros lugares de la Península, nunca antes habían sido mencionadas entre las monoflorales producidas en Galicia. Sin embargo, este taxón ha aparecido con porcentajes relevantes en algunas mieles milflores o de otra monofloralidad, de producción artesanal gallega (Díaz et al. 1997, de Sá-Otero et al. 2009, 2011). Según Schweitzer (2013) esta clase de mieles poseen características acidimétricas que las diferencian de las demás, siempre con un $\mathrm{pH}$ superior a 6, además de una gran pureza de monofloralidad (poca diversidad de fuentes de néctar dado el gran porcentaje que alcanza esta especie en el espectro polínico). Todas las muestras estudiadas tienen en común presentar espectros poco diversos, 3-12 tipos polínicos diferentes por miel (Tabla 1), en la mayoría de los casos uno o dos tipos polínicos representan el 90\% (Rhamnus frangula y Castanea sativa, excepcionalmente Rubus ulmifolius-t.) de la aportación polínica y todos los demás están en proporciones más bajas (Tabla 1).

Las mieles de Rhamnus frangula. Han resultado ser monoflorales de Rhamnus frangula siete, las identificadas con los números de muestra 1, 4, 6, 9, 12, 14 y 15. Todas ellas muy semejantes entre sí, excepto las 14 y 15, todas las demás llevan como polen más abundante, después de Rhamnus frangula, el de Castanea sativa. El de Rubus ulmifolius-t. es también común a todas ellas, excepto para las muestras 1 y 14. Otros pólenes presentes son el de Reseda media, Jasione montana, Prunus spinosa-t. y Conium maculatum. Su riqueza polínica es baja, clases de Maurizio I, II. La mayor riqueza de la muestra 14, clase Maurizio IV pudo deberse a una mayor aportación de granos de polen de castaño. La diversidad en tipos polínicos presentes oscila entre 12, en la miel número 9 y 3 , en la 14. Como no existe reglamentación en la IPG (Indicación Geográfica Protegida) para esta clase de mieles monoflorales se ha considerado como requerimiento mínimo un porcentaje mayor del $45 \%$ en polen de Rhamnus frangula, y se propone como combinaciones polínicas mayoritarias: Rhamnus frangula-Castanea sativa y Rhamnus frangula-Prunus spinosa-t.

Las mieles de Castanea sativa. Las monoflorales de Castanea sativa han sido las muestras número 7 y 8 , que han alcanzado porcentajes de polen de esta especie superiores al 80\%, de baja riqueza polínica (clase Maurizio I, la 7 y clase III, la 8). La diversidad de tipos polínicos es 
J. Rodríguez-Castiñeira et al. Origen botánico y contenido en proteína de mieles artesanales procedentes de colmenares...

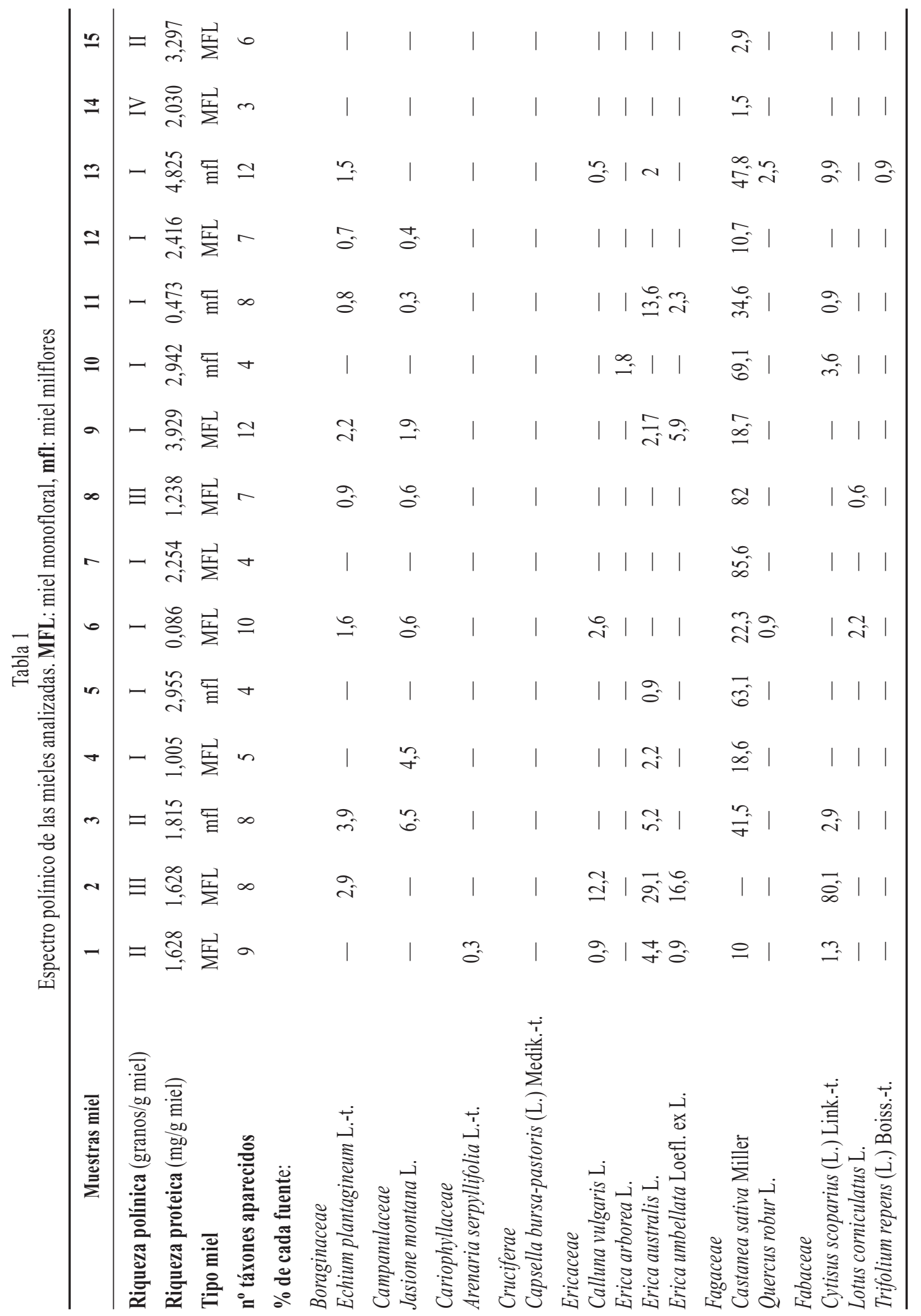


J. Rodríguez-Castiñeira et al. Origen botánico y contenido en proteína de mieles artesanales procedentes de colmenares...

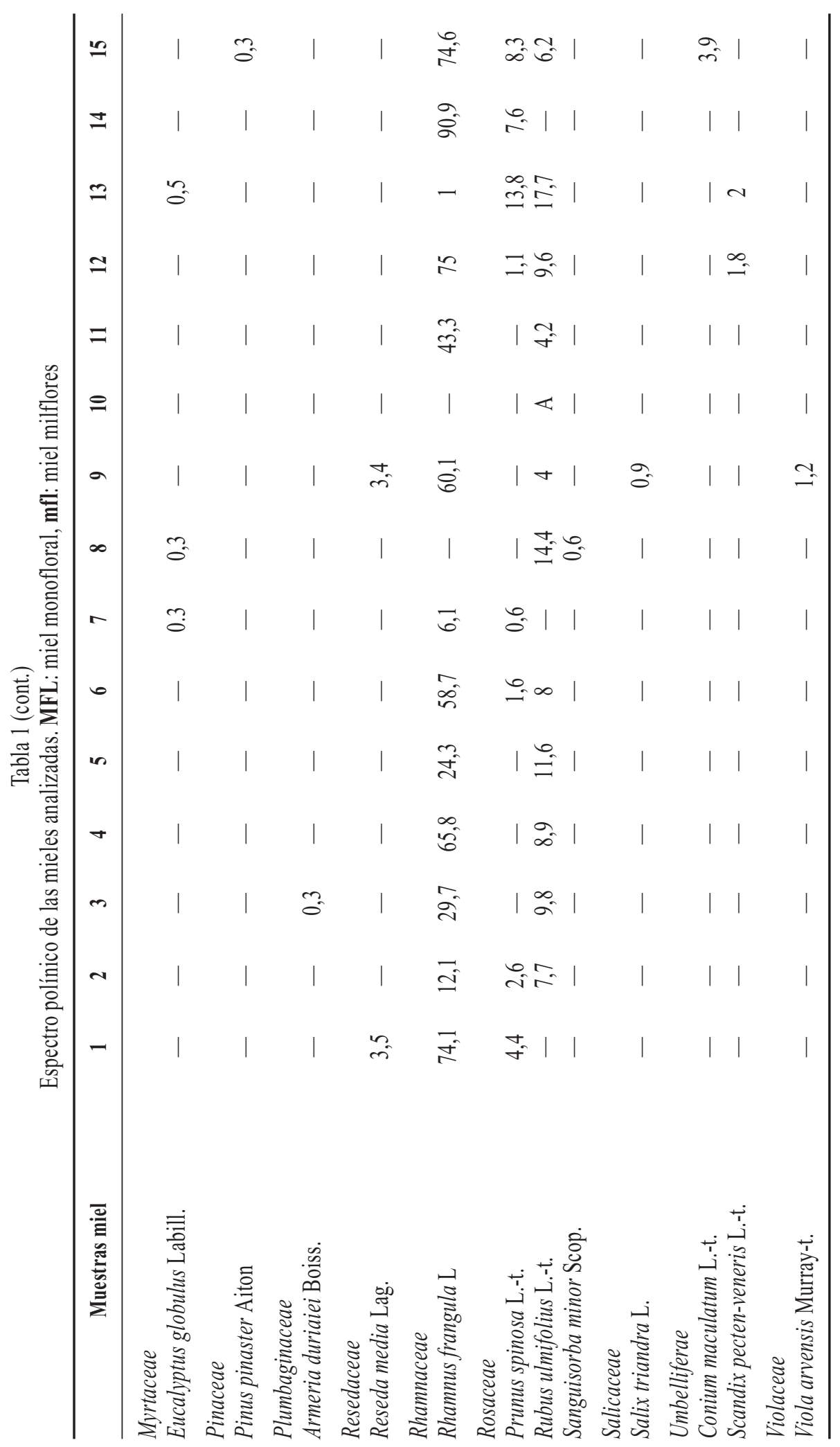


también muy baja, 4 y 7 tipos polínicos diferentes, respectivamente (Tabla 1). Por lo que se refiere a su espectro polínico, en la número 7 el polen más abundante, además de Castanea sativa, ha sido Rhamnus frangula; en la muestra 8, el porcentaje de polen de Castanea sativa va seguido del de Rubus ulmifolius-t.; todos los demás tipos tienen valores inferiores a $1 \%$. El espectro polínico de ambas mieles es semejante al de otras mieles de la misma naturaleza, artesanales o comerciales, obtenidas en otras comarcas gallegas (de Sá-Otero et al. 2009). La producción de mieles de castaño en Galicia ha sido referenciada con anterioridad por Sánchez-Cunqueiro \& Sáenz-Laín (1982), de Sá-Otero et al. (1991, 2009), Díaz et al. (1997), Seijo et al. (1997), Vázquez-Rodríguez (2001), Graña-Rivas (2004), Pirruccio-Barrosa (2003), de cuyo espectro polínico forman parte importante los pólenes de Castanea sativa, Rubus ulmifolius-t., Cytisus scoparius-t. y Erica. Ocasionalmente, en proporción destacada según las localidades de procedencia, también se pueden encontrar pólenes de Raphanus raphanistrum-t., Eucalyptus, Trifolium repens o Campanula. El espectro polínico de las mieles que en este estudio han resultado ser monoflorales de castaño son semejantes a los de otras mieles de la misma naturaleza, artesanales o comerciales, obtenidas en otras comarcas gallegas, salvo la reducida diversidad de su espectro polínico y los valores de polen de Rhamnus frangula.

La miel de Brezo. La miel $n^{0} 2$ es monofloral de brezo, en ellas la suma de porcentajes correspondientes a Erica australis, E. umbellata y Calluna vulgaris ha superado el $45 \%$ del total. Otras especies visitadas por la abeja para la elaboración de esta miel han sido Cytisus scoparius-t., Rhamnus frangula y Rubus ulmifolius-t., con por- centajes de polen en la clase de frecuencia I y Echium plantagineum-t. y Prunus spinosa-t., en la clase R. Su riqueza polínica es media, correspondiendo a la clase III de Maurizio. El número de tipos polínicos distintos es de 8 . De Sá-Otero et al. (2006), para las mieles monoflorales de brezo del Parque Natural de 0 Xurés, proponen como combinación mayoritaria para las mieles de brezo estudiadas la formada por los pólenes de Erica-t.-Cytisus scopariust.-Quercus robur-Castanea sativa. En dichas mieles de brezo, alcanzan valores porcentuales importantes los pólenes de Quercus robur y Castanea sativa. Seijo (1994) encuentra en las mieles de brezo producidas en distintas zonas de Galicia, como tipos polínicos secundarios más importantes: Castanea sativa, Rubus, Cytisus scopariust. y Eucalyptus, y de forma puntual tienen importancia Salix, Quercus, Centaurea y Calluna vulgaris.

Las mieles milflores. El grupo de las mieles milflores estudiadas está formado por cinco muestras; de éstas, cuatro tienen como fuente mayoritaria Castanea sativa. La muestra $n^{0} 11$ es una miel milflores con un claro predominio de Rhamnus frangula, rayando la clase $\mathrm{D}$, junto a Castanea sativa y al conjunto de las Ericas (Erica australis y E. umbellata) como tipos polínicos clase A; Rubus ulmifolius-t. aparece con un 4,25\% del espectro total. En total reúne néctar de 8 fuentes distintas que, a pesar de ser una diversidad media, han aportado una bajo número de granos de polen a la miel (su riqueza polínica es de las más pobres, 80 granos de polen/g de miel, correspondiendo a la clase I de Maurizio). Las mieles 3, 5, 10 y 13 son milflores en las que el polen de castaño ha sido el más abundante. Su espectro polínico es diverso, en las mieles 5,10 y 13 Castanea sativa alcanza valores de polen D, particularmente en la miel $n^{0} 10$, sus valores son casi los re-

Tabla 2

Relación riqueza proteica vs. variables diversas bajo el punto de vista de un análisis de componentes principales (González-Vázquez 2009)

\begin{tabular}{lcccc}
\hline \multicolumn{1}{c}{ \% de varianza explicado } & Eje I & Eje II & Eje III & Eje IV \\
\hline \multicolumn{1}{c}{$83^{\prime} 6$} & 29,7 & 23,6 & 18,2 & 12,1 \\
\hline Variables de la matriz de datos & & Porcentajes de inercia & \\
\hline Riqueza polínica & 0,755 & & & \\
Castanea sativa & 0,751 & & & \\
Rhamnus frangula & 0,660 & & & \\
Cytisus scoparius -t. & & 0,652 & & \\
Rubus ulmifolius-t. & & & 0,767 & \\
Erica sp. & & & & 0,085 \\
Monofloralidad & & & & \\
\hline
\end{tabular}


queridos para considerarla como monofloral. En las mieles 3 y 5 Rhamnus frangula se presenta como polen acompañante; en las $n^{0} 10$ y 13 , el polen acompañante es el de Rubus ulmifolius-t. Otros pólenes de interés en el espectro son Cytisus scoparius-t., Echium vulgare-t., Erica australis, Jasione montana, Prunus spinosa-t. La riqueza de fuentes de néctar de este grupo de mieles varía desde 4 (muestras 5 y 10) a 12, la máxima aparecida en este estudio (muestra 13); y casi todas pertenecen a la clase I de Maurizio (la miel $n^{0} 3$, a la clase II) en cuanto a riqueza polínica. Louveaux \& Vergeron (1964) señalan la complejidad en el espectro polínico de esta clase de mieles. En la región gallega dominan mieles mil flores cuya composición es diferente, según el lugar de producción. En la costa, muestran frecuencias elevadas de polen de Eucalyptus; las del interior, son ricas en polen de Castanea sativa, Rubus y Erica. Es posible apreciar una franja de confluencia en la que las mieles poseen características mixtas (Sanchez-Cunqueiro \& Saenz-Laín 1982, Terradillos 1988, Aira et al. 1990, Seijo 1994, Díaz 1995, Díaz et al. 1997, De Sá-Otero et al. 2009). Las mieles milflores que se han analizado en este trabajo se caracterizan por la reducida diversidad de su espectro polínico y muestran como característica muy particular los altos valores de polen de Rhamnus frangula que se alcanzan en algunas de ellas.

Importancia de los tipos polínicos aparecidos en las mieles estudiadas. La ordenación de los resultados del análisis polínico cualitativo en clase de frecuencia según Louveaux et al. (1978), es el modo de expresar la importancia relativa que el néctar procedente de las diversas especies de plantas libadas por la abeja, tiene en la elaboración de la miel. Rhamnus frangula se muestra como una fuente de néctar de primer orden, puesto que, de las 15 mieles estudiadas, el polen de sanguiño se muestra como dominante en siete, acompañante en cinco, e importante en tres (Tabla 1). En un único caso aparece formando parte de las clases de frecuencia polen raro o presente ( $\mathrm{P}$ en la muestra 13). Castanea sativa es también fuente importante de néctar y se halla como polen más abundante en seis de las mieles, como segunda especie más frecuentada, en seis. Rubus ulmifolius-t. es también fuente de néctar de preferencia para la abeja, puesto que forma parte de la clase de frecuencia acompañante, y es la segunda 0 tercera especie más frecuentada en diez de las mismas. Las plantas que integran los demás tipos polínicos presentes en los espectros polínicos de las mieles, aparecen en las mismas de forma ocasional, su néctar participa, por tanto, de forma minoritaria en la elaboración de éstas. Alguna de ellas puede tener importancia destacada en mieles concretas.
Para este grupo de mieles artesanales estudiadas, dada la peculiaridad de las mieles monoflorales de Rhamnus frangula, se pueden proponer como pólenes en la clase de frecuencia D (dominantes) a Castanea sativa, Erica ssp. $y$ Rhamnus frangula; en la clase de frecuencia A (acompañantes) a Castanea sativa, Erica australis, E. umbella, Rhamnus frangula y Rubus ulmifolius-t. Como clase I (importante) a Calluna vulgaris, Castanea sativa, Cytisus scoparius-t., Conium maculatum, Echium vulgare-t., Erica australis, E. umbellata, Rhamnus frangula, Jasione montana, Prunus spinosa-t., Reseda media y Rubus ulmifolius-t. Como clase $\mathrm{R}$ (polen raro) a Calluna vulgaris, Castanea sativa, Cytisus scoparius-t., Echium vulgare-t., Erica arborea, E. australis, E. umbellata, Jasione montana, Prunus spinosa-t., Quercus robur-t., Scandix pectenveneris, y Viola arvensis Murray-t. Como clase P (presente) Arenaria serpyllifolia -t., Armeria duriaei, Calluna vulgaris, Capsella bursa-pastoris-t., Cytisus scoparius, Echium vulgare, Erica australis, E. umbellata, Eucalyptus globulus, Rhamnus frangula, Jasione montana, Lotus corniculatus, Pinus pinaster, Prunus spinosa-t., Quercus robur, Salix triandra, Sanguisorba minor, Trifolium repens-t.

Análisis de proteínas. Con la referencia de que la mayor parte de las proteínas existentes en la miel derivan del polen contenido en la misma (Crane 1975), se ha establecido una relación entre la riqueza proteica de cada muestra analizada y su espectro polínico. Alguna de las mieles muestra valores de proteína más altos que los obtenidos por otros autores (Fonseca et al. 1993). Sá-Otero et al. (2000) encontraron en el polen apícola producido en colmenares de Galicia valores relativamente altos de proteína, atribuibles a la abundancia del polen de Cytisus scoparius-t. en el producto. González-Vázquez (2009) estudió la relación entre la riqueza proteica y los parámetros de monofloralidad, riqueza polínica y aparición e importancia de diversas fuentes de néctar, a través de un análisis de Componentes Principales, proponiendo que el contenido en proteínas de las mieles estaba condicionado por dichas variables, de modo que los componentes que más influían en la variación de la riqueza proteica eran la riqueza polínica y la presencia de Castanea sativa, Rhamnus frangula, Cytisus scoparius-t., Rubus ulmifolius-t. y Erica sp. Resultados semejantes han sido aportados por Sande-González (2004) y Castiñeiras-Fernández (2005).

En el presente estudio se ha calculado el coeficiente de correlación (estadístico r de Pearson) entre el valor proteico de cada muestra de miel y las diversas variables propuestas por los autores antes mencionados, riqueza polínica, monofloralidad y cada una de las fuentes de néctar, 
J. Rodríguez-Castiñeira et al. Origen botánico y contenido en proteína de mieles artesanales procedentes de colmenares...

Tabla 3

Valores del coeficiente $\mathrm{r} 0$ de Pearson para los distintos parámetros comparados (riqueza proteica con otras características de la miel analizadas, considerando el total de las mieles y en los diferentes grupos de mieles de la misma naturaleza). $\square$ : relación directa, $\diamond$ : relación inversa; gradación de intensidad de la relación: 1-0,8, (4 símbolos); 0,8-0,6 (3 símbolos); 0,6-0,4 (2 símbolos); 0,4-0,2, intensidad muy suave (1 símbolo); valores de 0,2 a $-0,2$ han sido considerados no significativos $(\varnothing)$

\begin{tabular}{|c|c|c|c|c|}
\hline & Global & $\begin{array}{c}\text { Frangula } \\
\text { dominante }\end{array}$ & $\begin{array}{c}\text { Castanea } \\
\text { dominante }\end{array}$ & $\begin{array}{l}\text { Milflores } \\
\text { varias }\end{array}$ \\
\hline Riqueza Polínica & $\varnothing$ & $\varnothing$ & $\diamond \diamond \diamond$ & $\diamond$ \\
\hline Monofloralidad & $\varnothing$ & & & \\
\hline Diversidad fuentes néctar & $\varnothing$ & $\varnothing$ & प्व & $\square$ \\
\hline Armeria duriaei & $\varnothing$ & & & $\diamond$ \\
\hline Arenaria serpyllifolia-t. & $\varnothing$ & $\varnothing$ & & \\
\hline Calluna vulgaris & $\diamond$ & $\diamond \diamond \diamond$ & $\square \square \square \square$ & $\diamond$ \\
\hline Capsella bursa-pastoris-t. & $\emptyset$ & $\square \square \square$ & & \\
\hline Castanea sativa & $\varnothing$ & $\diamond \diamond$ & & $\square \square$ \\
\hline Conium maculatum & $\square$ & $\square \square$ & & \\
\hline Cytisus scoparius-t. & $\square$ & $\diamond \diamond$ & $\diamond \diamond \diamond \diamond$ & $\square$ \\
\hline Echium plantagineum-t. & $\emptyset$ & $\varnothing$ & $\square$ & $\square$ \\
\hline Erica arborea & $\emptyset$ & & $\varnothing$ & \\
\hline Erica australis & $\diamond$ & $\varnothing$ & $\square \square \square \square$ & $\diamond \diamond$ \\
\hline Erica umbellata & $\emptyset$ & प्वप & & $\diamond$ \\
\hline Eucalyptus globulus & $\square$ & & $\square$ & $\square \square \square$ \\
\hline Rhamnus frangula & $\varnothing$ & $\varnothing$ & $\varnothing$ & $\diamond \diamond \diamond$ \\
\hline Graminea silvestre & $\square$ & प्र & & \\
\hline Jasione montana & $\varnothing$ & $\varnothing$ & $\diamond \diamond \diamond$ & $\diamond$ \\
\hline Lotus corniculatus & $\diamond \diamond$ & $\diamond \diamond \diamond$ & $\diamond \diamond \diamond$ & \\
\hline Pinus pinaster & $\square$ & $\square \square$ & & \\
\hline Prunus spinosa-t. & $\square \square$ & $\square$ & प्म० & $\square \square \square$ \\
\hline Rubus ulmifolius-t. & $\square$ & $\varnothing$ & $\square$ & $\square \square \square$ \\
\hline Quercus robur-t. & $\square$ & $\diamond \diamond \diamond$ & प्व०० & $\square \square \square$ \\
\hline Reseda media & $\varnothing$ & $\square$ & & \\
\hline Salix triandra & $\square$ & प्व & & \\
\hline Sanguisorba minor & $\diamond$ & & $\diamond \diamond \diamond$ & \\
\hline Scandix pecten-veneris-t. & $\square \square$ & $\varnothing$ & प्वप & $\square \square \square$ \\
\hline Trifolium repens-t. & $\square \square$ & & वप्व & $\square \square \square$ \\
\hline Viola arvensis-t. & $\square$ & प्व & & \\
\hline
\end{tabular}

respectivamente (Tabla 3). La relación de la riqueza proteica con la riqueza polínica, con el carácter monofloral y con la diversidad de fuentes no parece significativa, cuando se comparan entre sí todas las mieles. Por tanto, para analizar esta propiedad es necesario comparar grupos de naturaleza más homogénea con un origen floral semejante, por ejemplo. Así, si la comparación se realiza por grupos naturales de mieles en relación a su origen floral, es decir; mieles de Frangula dominante, Castanea sativa dominante 0 milflores varías, puede verse: a) riqueza proteica frente a características propias del grupo (riqueza polínica y diversidad de fuentes). En este caso la relación es inexistente para mieles de Frangula dominante; para las de Castanea dominante, es directa con riqueza polínica e inversa con respecto a diversidad de fuentes; si se trata de mieles milflores varias se da una relación semejante pero 
de menor intensidad (Tabla 3). b) riqueza proteica frente a los diferentes tipos polínicos. Se muestran diferentes grados de relación para los grupos de mieles considerados.

La valoración del contenido en proteínas frente a la presencia de polen de Castanea sativa en la miel, muestra una relación directa cuando se trata de mieles milflores, inversa para las mieles de Frangula y Castanea. Si este parámetro se valora frente a la presencia de polen de Rhamnus frangula en la miel, la relación es inversa en el caso de mieles milflores e inexistente en los demás supuestos. Si se valora frente a la presencia de polen de Echium plantagineum-t. la relación es directa para las mieles de Castanea dominante y milflores, indirecta en los demás casos. Para la presencia de polen de Cytisus scoparius-t, Eucalyptus globulus, Prunus spinosa-t., Quercus robur-t., Rubus ulmifolius-t., Scandix pecten-veneris-t .y Trifolium repens-t. la relación es positiva, con intensidad variable, para los diferentes grupos naturales de mieles, excepto en caso de mieles de Rhamnus frangula L. dominante, que es inexistente para la presencia de Rubus ulmifolius-t. y Scandix pecten-veneris-t e inversa para Quercus robur-t. y Cytisus scoparius-t. La valoración del parámetro frente a la presencia de pólenes de Jasione montana y Calluna vulgaris es inversa o inexistente, salvo para el caso de $C$. vulgaris en mieles de Castanea dominante. Para el caso de la presencia de polen de Reseda media la relación es directa en las mieles de Rhamnus frangula (al igual que Conium maculatum, gramínea silvestre, Pinus pinaster, Salix triandra y Viola arvensis-t.).

En síntesis, el contenido en polen de Castanea sativa presenta una relación inversa con la riqueza proteica en las mieles en las que Rhamnus o Castanea son polen dominante, mientras que en las mieles milflores es directa. El contenido en polen de Rhamnus frangula presenta una relación inversa muy alta en el grupo de las mieles milflores, y en el resto sus valores no son significativos. El contenido en polen de Cytisus, Eucalyptus, Prunus, Quercus, Rubus y Scandix presentan una relación directa en los gru- pos «todas las mieles», Castanea dominante y milflores. El de Lotus corniculatus presenta una relación inversa con la riqueza proteica en casi todos los casos.

\section{CONCLUSIONES}

Por primera vez se obtienen mieles de tan alto porcentaje en polen de Rhamnus frangula para la Península Ibérica y para Galicia. Estas tienen como particularidad la baja diversidad de su espectro polínico. Un contenido en proteína muy variable. Las mieles más ricas han sido las de Castanea dominante, seguidas de las milflores.

Los taxones que más han contribuido al valor de la riqueza proteica cuando las mieles son consideradas en su totalidad, han resultado ser: a) con relación directa, Prunus spinosa-t., Scandix pecten-veneris-t. y Trifolium repens-t. b) con relación inversa, Lotus corniculatus.

Los taxones que más han contribuido al valor de la riqueza proteica en las mieles con Rhamnus frangula dominante, han resultado ser: a) con relación directa, Capsella bursa-pastoris-t., Erica umbellata, gramínea silvestre, Salix triandra y Viola arvensis-t. b) con relación inversa $\mathrm{Ca}$ lluna vulgaris, Lotus corniculatus y Quercus robur-t.

Los taxones que más han contribuido al valor de la riqueza proteica en mieles con Castanea dominante, han resultado ser: a) con relación directa, Calluna vulgaris, Cytisus scoparius-t., Erica australis, Prunus spinosa-t., Quercus robur-t., Scandix pecten-veneris-t. y Trifolium repens-t. b) con relación inversa Castanea sativa, Jasione montana, Lotus corniculatus y Sanguisorba minor.

Los taxones que más han contribuido al valor de la riqueza proteica en las mieles Milflores han resultado ser: a) con relación directa, Eucalyptus globulus, Prunus spinosa-t., Rubus ulmifolious-t., Quercus robur-t. y Scandix pecten-veneris-t. y Trifolium repens-t. b) con relación inversa Rhamnus frangula.

\section{BIBLIOGRAFÍA}

Aira, M. J.; Ramil-Rego, P. \& SÁ-Otero, M. P. 1990. Identificación polínica de Ericaceae en mieles gallegas. Acta Bot. Malacitana 15: 27-32.

Azeredo, L. C.; Azeredo, M. A.; De Sousa, S. R. \& Dutra, V. M. L. 2003. Protein contents and physicochemical properties in honey simples of Apis mellifera of different floral origins. Food Chem. 80(2): 249-254.

Baroni, M. V.; Chiabrando, G. A.; Costa, C. \& Wunderlin, D. A. 2002. Assessment of the floral origin of honey by SDS-Page Inmunoblot Techiques. J. Agric. Food Chem. 50: 1362-1367.
BRADFORD, M. 1976. A rapid and sensitive method for the quantification of microgram quantities of protein utilizing the principle of protein-dye binding. Anal. Biochem. 172: 248-54.

Castiñeiras-Fernández, S. 2005. Contenido proteico de mieles florales con denominación de calidad «Mel de Galicia». Proyecto Fin de Carrera. Universidad de Vigo, Facultad de Ciencias de Ourense.

Crane, E. 1975. Honey: a comprehensive survey. Russak \& Co. Inc, New York.

Cremonez, T. M.; Jong, D. \& Botondi, M. M. G. 1998. Quantification of hemolymph proteins as a fast method for tes- 
J. Rodríguez-Castiñeira et al. Origen botánico y contenido en proteína de mieles artesanales procedentes de colmenares...

ting protein diets for honey bees (Hymenoptera: Apideae). J. Econ. Entomol 91(16): 1284-1289.

De Sá-Otero, M. P.; Armesto-Baztán, S. \& Díaz-Losada, E. 2006. A study of variations in the pollen sampled from a small geographical area in NW Spain (Baixa Limia-Serra do Xurés Nature Reserve). Grana 45: 137-45.

De Sá-Otero, M. P.; Armesto-Baztán, S. \& Díaz-Losada, E. 2009. Espectro polínico de mieles de castaño, Castanea sativa Miller (1768), (Fagaceae, Fagales) de la comarca geopolítica de Allariz-Maceda (Ourense, NO España). Bol. R. Soc. Esp. Hist. Nat. Séc. Biol. 103(1-4): 27-35.

De Sá-Otero, M. P.; Armesto-Baztán, S. \& Díaz-Losada, E. 2011. Caracterización de mieles de obtención artesanal y comerciales producidas en Galicia (NO España) a partir de su espectro polínico y contenido proteico. Bot. Complut. 35: $131-140$.

Díaz, E. 1995. Aportación del conocimiento del origen botánico y características físico-químicas del polen apicola en Galicia. Tesis Doctoral, Facultad de Ciencias de Ourense. Universidad de Vigo.

DíAz, E.; GónZALEZ, A.V.; SÁa-OTERO, M.P. 1997. Estudio melisopalinológico en Galicia (NO de España). Orsis 12: 27-38.

FonseCA, E.; KleinerT, A. \& Amaral, A. D. 1993. Meis Brasileiros: estudio de su origen floral. Apicultura no Brasil 16(5): 30-33.

GHeLDOF, N.; WANG, X. \& ENGESETh, N. J. 2002. Identification and quantification of antioxidant components of honeys from variousfloral sources. J. Agric. Food Chem. 50: 58705877.

GonzáLEZ-VÁzquez, G. 2009. Determinación de parámetros de calidad, naturaleza, procedencia floral, riqueza polínica y contenido en proteina; en mieles artesanales de las comarcas geopoliticas ourensanas de "Terra de Caldelas», «O Ribeiro" y otras. Proyecto Fin de Carrera, Facultad de Ciencias de Ourense, Universidad de Vigo.

GRAÑA-RIVAS, M. F. 2004. Certificación de origen floral y geográfico en mieles comercializadas con etiqueta «Miel de Galicia». Proyecto Fin de Carrera, Facultad de Ciencias de Ourense, Universidad de Vigo.

Louveaux, J. \& Vergeron, P. 1964. Etude du spectre pollinique de quelques miels espagnols. An. Abeille 7(4): 329-347.

LouveauX, J.; Maurizio, A. \& VorwoHL, G. 1978. Methods of melissopalynology. Bee World 54 (4): 139-157.

MauRIZIO, A. 1939. Untersuchungen zur quantitativen pollen analyse des honings. Mitt. Get. Lebensmnitelterr: 30(1-3): 27-69.

MoORE, P. D. \& WEBB, J.A. 1991. An illustrated guide to pollen analysis. Hodder \& Stoughten, London.
Padovan, G. J.; De Jong, D.; Rodrigues, L.P. \& Marchini, J.S. 2003. Detection of adulteration of commercial honey samples by the ${ }^{12} /{ }_{13} \mathrm{C}$ ratio. Food Chem. 82: 633-636.

Pirruccio-Barrosa, M. C. 2003. Aportación al conocimiento de la producción melifera en la comarca geopolíica «Maceda-Allariz». Proyecto Fin de Carrera. Facultad de Ciencias de Ourense, Universidad de Vigo.

Sáa-Otero, M. P.; Ramil-Rego, P. \& Aira-Rodríguez, M. J. 1991. Análisis polínico de mieles procedentes de las provincias de Lugo y Orense (Galicia, España). Nova Ac. Cient. Compostelana (Bioloxía) 2: 57-63.

Sáa-Otero, M. P.; Díaz-Losada, E. \& González-Porto, A. V. 1993. Estudio estadístico de representatividad de los datos obtenidos en análisis polínico de mieles de Orense (España). Bol. R. Soc. Esp. Hist. Nat. (Sec. Biol.) 90(1-4): 5-16. Sáa-Otero, M. P.; Suárez-Cervera, M. \& Gracia, V. R. 1996. Atlas de polen de Galicia. I. Diputación Ourense, Ourense.

SÁa-Otero, M. P.; Díaz-Losada E. \& Fernández-Gómez, E. 2000. Analysis of fatty acids, proteins and ethereal extract in honeybee pollen. Grana 39: 175-181.

Sanchez-Cunqueiro, C. \& Sáenz-Laín, C. 1982. Análisis polínico de mieles de Pontevedra (España). Lazaroa 4: 253-268.

SANDE-GonZáLez, L. 2004. Análisis del contenido proteico de mieles florales de producción artesanal y comercial. Proyecto Fin de Carrera. Facultad de Ciencias de Ourense, Universidad de Vigo.

SchweitZe, P. 2013. Terre des hommes, planète du miel. Les miels monofloraux: la bourdaine. L'Abeille de France \& L'Apiculteur 1008 (décembre).

Seijo-Coello, M. C. 1994. Caracterización de la miel de Galicia a través del espectro polínico. Tesis Doctoral. Universidad de Vigo.

Seijo, M. C.; Jato, M. V.; Aira, J. \& Iglesias, I. 1997. Uniflorals honeys of Galicia (North-West Spain). J. Apicult. Res. 36(3-4): 133-139.

TerRadillos, L. A. 1988. Estudio microscópico del sedimento de la miel y su aplicación en la caracterización de mieles de Galicia. Tesis Doctoral. Facultad de Farmacia, Universidad de Santiago.

Valdés, B.; Díez, M. J. \& Fernández, I. 1987. Atlas polínico de Andalucía occidental. Inst. Desar. Reg., Dip. Cádiz, Sevilla.

VÁzquez-Rodríguez, A. 2001. Caracterización de las mieles del Parque Natural «O Xurés». Proyecto Fin de Carrera. Facultad de Ciencias de Ourense, Universidad de Vigo. 